Journal of Engineering and Applied Sciences 7 (5): 363-371, 2012

ISSN: 1816-949X

(C) Medwell Journals, 2012

\title{
Modelling and Experimental Validation of a Passive Scalar Diffusion
}

\author{
${ }^{1}$ D. Tcheukam-Toko, ${ }^{1}$ C.A. Koueni-Toko, ${ }^{1}$ R. Mouangue and ${ }^{2}$ P. Paranthoen \\ ${ }^{1}$ Department of Energetic Engineering, IUT, \\ University of Ngaoundere, P.O. Box 455 NDere, Cameroon \\ ${ }^{2}$ CNRS UMR 6614 CORIA, University of Rouen, P.O. Box 12-76801, \\ Saint-Etienne du Rouvray, France
}

\begin{abstract}
The atmospheric pollution is one of the most dominating phenomenons now-a-days. It is generated partially by warm gases emitted by transporting vehicles and industrial activities and equally has origin the degradation of the environment in general and the quality of air in particular. This study concerns the homogenous compressible flows with constant viscosity. It consist of an experimental validation and modelling of the diffusion of a passive scalar in an interacting turbulent flow with two heated oblique air jets inclined at an angle of $45^{\circ}$ in relation to the principal direction. The two jets emission holes are circular with a diameter of $5 \mathrm{~mm}$ and spaced out by $40 \mathrm{~mm}$. This study treats the influence of the initial velocities of jets on the diffusion of the temperature field. The problem was tackled using the calculation codes of Gambit-Fluent to simulate the diffusion of a scalar. The results show that the flow temperature decreases with the increase of the Reynolds number and the scalar sustains lesser and lesser influence on the longitudinal flow. The numerical results are in account with the experimental results.
\end{abstract}

$\underline{\text { Key words: Passive scalar, diffusion, jets, modelling, turbulent flow, France }}$

\section{INTRODUCTION}

The atmospheric pollution is generated partially by warm gases emitted by transporting vehicles and industrial activities. If we wish to know the impact of the pollution on the environment and the public health and act with much effectiveness, it is necessary to study these pollutants from their emission sources to their dispersion in the environment. This brings the study mixture phenomenon and the dispersion of scalars.

In this study, researchers are treating the phenomenon of the dispersion of passive scalar in a turbulent flow of homogenous compressible fluid at constant viscosity and density. Numerous studies had been realised on these phenomenon, precisely that of Gosse et al. (2006a, b) on the dispersion of a passive scalar in the near wake of the Ahmed bodies applied to the automobile pollution which shows the presence of whirl in the flow modifies the dispersion of the field of temperature. The studies of Jordan shows that the presence of whirl in the flow modifies in an important manner the velocity field and reduces the concentration of pollutants. Vincont et al. (2000) shows that the dispersion of a passive scalar was possible in a turbulent boundary layer generated by an obstacle. Kothnur and
Clemens (2005) shows that the propagation direction of the scalar is perpendicular to that of the share stress. Researchers notice that the most part of this research had been done in the presence of obstacles in the flow. Very little studies had taken note of the inclination of air jets. Bray and Garry (1999) had carried out an experimental study on the optimisation of the generation of vortex for air jets.

The jets are inclined at $30^{\circ}, 45^{\circ}$ and $60^{\circ}$. It shows that the jets inclined at an angle of $45^{\circ}$ permits a good quality of vortex. The aim of this study is to do a numerical modelling of the evolution of the temperature field so as to compare the obtained results to experience ones. In order to better carry out this study, researchers are going to present the mathematical models used followed by the experimental device and the results obtained and at the end, compare its numerical results to experience ones and conclude.

\section{MATERIALS AND METHODS}

The continuity equation is given by Eq. 1:

$$
\frac{\partial \rho}{\partial \mathrm{t}}+\operatorname{div}(\rho \overrightarrow{\mathrm{V}})=0
$$

Corresponding Author: D. Tcheukam-Toko, Department of Energetic Engineering, IUT, University of Ngaoundere, P.O. Box 455 NDere, Cameroon 
The conservation equations of the average quantity of movement of Navier-Stockes known by the name RANS are for compressible fluid and Newtonian given by Eq. 2:

$$
\begin{aligned}
& \frac{\partial}{\partial t}\left(\rho u_{i}\right)+\underbrace{\frac{\partial}{\partial x_{j}}\left(\rho u_{i} u_{j}\right)}_{\text {Convective transport }}=-\underbrace{\frac{\partial \rho}{\partial x_{i}}}_{\substack{\text { Fores } \\
\text { duet } \\
\text { pressions }}}+\underbrace{\frac{\partial}{\partial x_{j}}\left[\mu\left(\begin{array}{l}
\frac{\partial \mu_{i}}{\partial x_{j}}+\frac{\partial \mu_{j}}{\partial x_{i}}- \\
\frac{2}{3} \delta_{i j} \frac{\partial \mu_{i}}{\partial x_{j}}
\end{array}\right)\right]}_{\text {Vis cosity forces }}+ \\
& \underbrace{\frac{\partial}{\partial \mathrm{x}_{\mathrm{j}}}\left(-\overline{\rho_{\mathrm{i}}^{\prime} \mathrm{u}_{\mathrm{j}}^{\prime}}\right)+\mathrm{F}_{\mathrm{i}}}_{\text {Forces generated by turbulence }}
\end{aligned}
$$

$-\overline{\rho u_{i}^{\prime} u^{\prime}}$ are the components of the Reynolds stress. Its expression is bellow as given by the Boussinesq's hypothesis:

$$
-\overline{\rho u_{i}^{\prime} u_{j}^{\prime}}=\mu_{t}\left(\frac{\partial u_{i}}{\partial x_{j}}+\frac{\partial u_{j}}{\partial x_{i}}\right)-\frac{2}{3}\left(\rho k+\frac{\partial u_{i}}{\partial x_{j}}\right) \delta_{i j}
$$

The k- $\varepsilon$ Turbulence Models used by the software FLUENT (3) are:

- The k- $\varepsilon$ Standard Model

- The k-e RNG Model

- The k-e Realisable Model

Researchers are going to use the k- $\varepsilon$ Realisable Model to carry out calculations in the software FLUENT. The turbulence k- $\varepsilon$ Realisable Model proposed by Shih et al. (1995) was proposed to make up for the insufficiency of the other k- $\varepsilon$ Models such as the k- $\varepsilon$ Standard Model, the k- $\varepsilon$ RNG Model, etc. by adopting a new formula for the turbulent viscosity while implicating a variable $\mathrm{C}_{\mu}$ at the origin (proposed by Reynolds) and a new equation for the disposed based on the dynamic equation of the vortices fluctuations. The equations of its transporting equations are: the turbulent kinetic energy transport equation which is given by Eq. 4 :

$$
\begin{aligned}
\frac{\partial}{\partial \mathrm{t}}(\rho \mathrm{k})+\frac{\partial}{\partial \mathrm{x}_{\mathrm{i}}}\left(\rho \mathrm{ku}_{\mathrm{j}}\right)= & \frac{\partial}{\partial \mathrm{x}_{\mathrm{i}}}\left[\left(\mu+\frac{\mu_{\mathrm{t}}}{\sigma_{\mathrm{k}}}\right) \frac{\partial_{\mathrm{k}}}{\partial \mathrm{x}_{\mathrm{j}}}\right]+ \\
& \mathrm{G}_{\mathrm{k}}+\mathrm{G}_{\mathrm{b}}-\rho \varepsilon-\mathrm{Y}_{\mathrm{M}}
\end{aligned}
$$

The transport equation of the dissipation rate of turbulent kinetic energy which is given by Eq. 5 :

Table 1: The constants of model
\begin{tabular}{llll}
\hline $\mathrm{C}_{\mathrm{mu}}$ & $\sigma_{\mathrm{k}}$ & $\sigma_{\varepsilon}$ \\
\hline 1.44 & 1.9 & 1.0 & 1.2 \\
\hline
\end{tabular}

$$
\begin{gathered}
\frac{\partial}{\partial t}(\rho \varepsilon)+\frac{\partial}{\partial \mathrm{x}_{\mathrm{i}}}\left(\rho \varepsilon \mathrm{u}_{\mathrm{j}}\right)=\frac{\partial}{\partial \mathrm{x}_{\mathrm{i}}}\left[\left(\mu+\frac{\mu_{\mathrm{t}}}{\sigma_{\varepsilon}}\right) \frac{\partial_{\varepsilon}}{\partial \mathrm{x}_{\mathrm{j}}}\right]+ \\
\rho \mathrm{C}_{1} \mathrm{~S} \varepsilon-\rho \mathrm{C}_{2} \frac{\varepsilon^{2}}{\mathrm{k}+\sqrt{\nu \varepsilon}}+\mathrm{C}_{1 \varepsilon} \frac{\varepsilon}{\mathrm{k}} \mathrm{C}_{3 \varepsilon} \\
\mathrm{C}_{1}=\max \left[0.43, \frac{\eta}{\eta+5}\right] \text { with } \eta=\mathrm{S} \frac{\mathrm{k}}{\varepsilon}
\end{gathered}
$$

Where:

$\mathrm{G}_{\mathrm{k}} \quad=$ The turbulent kinetic energy due to the average gradient of velocity

$\mathrm{G}_{\mathrm{b}} \quad=$ The generation of kinetic energy due to floating

$\mathrm{Y}_{\mathrm{M}} \quad=$ The contribution of the fluctuating dilatation

$\mathrm{C}_{2}$ and $\mathrm{C}_{1 \varepsilon}=$ The constants

$\sigma \mathrm{k}$ and $\sigma \varepsilon=$ The numbers of turbulent prandtl relative to $\mathrm{k}$ and $\varepsilon$

The values of constants are shown in Table 1 . The turbulent transport of heat is modelled by the usage of the analogy concepts of Reynolds to the turbulent transfer. The energy equation is given as:

$$
\frac{\partial}{\partial t}(\rho E)+\frac{\partial}{\partial x_{i}}\left[u_{i}(\rho E+P)\right]=\frac{\partial}{\partial x_{j}}\left(k_{e f f} \frac{\partial T}{\partial x_{j}}+u_{i}\left(\tau_{i j}\right)_{e f f}\right)+s_{h}
$$

$\mathrm{E}$ is the total energy, its expression is:

$$
\mathrm{E}=\mathrm{h}-\frac{\mathrm{P}}{\rho}+\frac{\mathrm{U}_{\mathrm{i}}^{2}}{2}
$$

$\mathrm{K}_{\text {eff }}$ is the coefficient of effective thermal conductivity and $\mathrm{K}$ is the coefficient of laminar thermal conductivity expressed as:

$$
\mathrm{k}_{\text {eff }}=\mathrm{k}+\frac{\mathrm{C}_{\mathrm{p}} \mu_{\mathrm{t}}}{\mathrm{Pr}_{\mathrm{t}}}
$$

$\left(\tau_{\mathrm{ij}}\right)_{\text {eff }}$ is the Tension Newtonian effective of vicious stress. Its expression is given by Eq. 9:

$$
\left(\tau_{\mathrm{ij}}\right)_{\mathrm{eff}}=\mu_{\mathrm{eff}}\left(\frac{\partial \mu_{\mathrm{j}}}{\partial \mathrm{x}_{\mathrm{i}}}+\frac{\partial \mathrm{u}_{\mathrm{i}}}{\partial \mathrm{x}_{\mathrm{j}}}\right)-\frac{2}{3} \mu_{\mathrm{eff}} \frac{\partial \mathrm{u}_{\mathrm{i}}}{\partial \mathrm{x}_{\mathrm{i}}} \delta_{\mathrm{ij}}
$$

Experimental device: This study is realised by means of a model shown in Fig. 1. The emission of pollutants is simulated by injecting hot air at a velocity $U_{j}$ by 


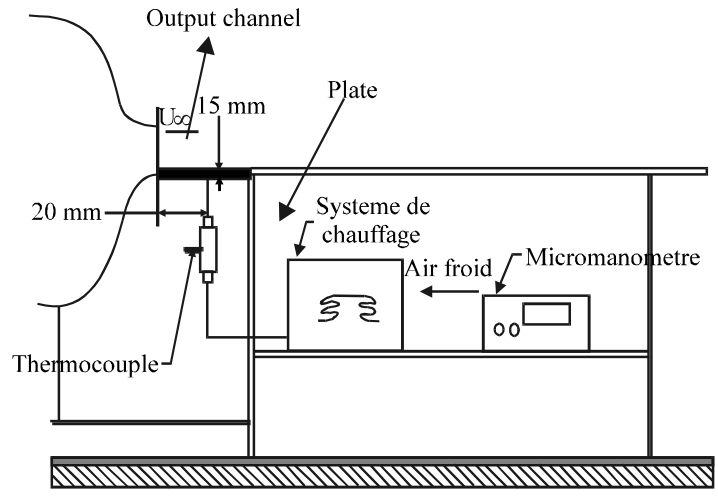

Fig. 1: Experimental device

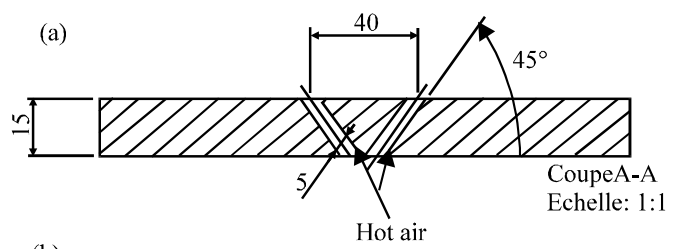

(b)

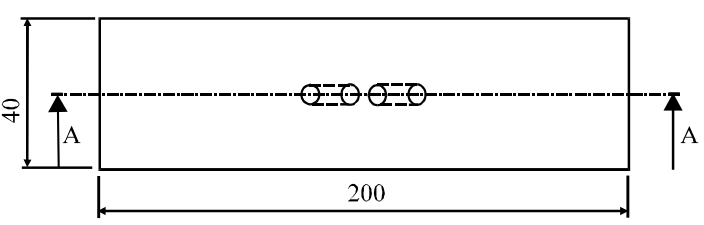

Fig. 2: Plate: a) Front view; b) Top view

intermediary of a small pipe with diameter $\mathrm{d}=3.5 \mathrm{~mm}$ placed on the back face of the model as shown in Fig. 1. The temperature gap between the jet and the exterior is maintained constant at $\Delta \mathrm{T}_{\text {ref }}=20^{\circ} \mathrm{C}$ by means of a regulated supply. The thermostat of the heating system is linked to the thermocouple of the regulation room. The small temperature gaps permits the resistance of gravity effects. The velocity $U_{j}$ of this heated flow is measured by means of a pressure lost linked to a micro manometer furness control.

The plate shown in Fig. 2 has a thickness of $15 \mathrm{~mm}$, a length of $200 \mathrm{~mm}$ and a width of $40 \mathrm{~mm}$. On this plate, researchers have two bored circular holes of diameter 5 $\mathrm{mm}$ inclined at $45^{\circ}$ in relation to the horizontal.

The two heated jets outlet of the nozzle across circular holes effectuated on a fixed plate at the level of the lower edge of the nozzle outlet. They are separated from one another by $40 \mathrm{~mm}$. The temperature fields have been analysed with the aid of a warm wire anemometer.

The calculation domain shown in Fig. 3 is a cobbled of length $200 \mathrm{~mm}$ of width $100 \mathrm{~mm}$ and of height $30 \mathrm{~mm}$. On this domain, the air jets (jet 1 and 2) are emitted

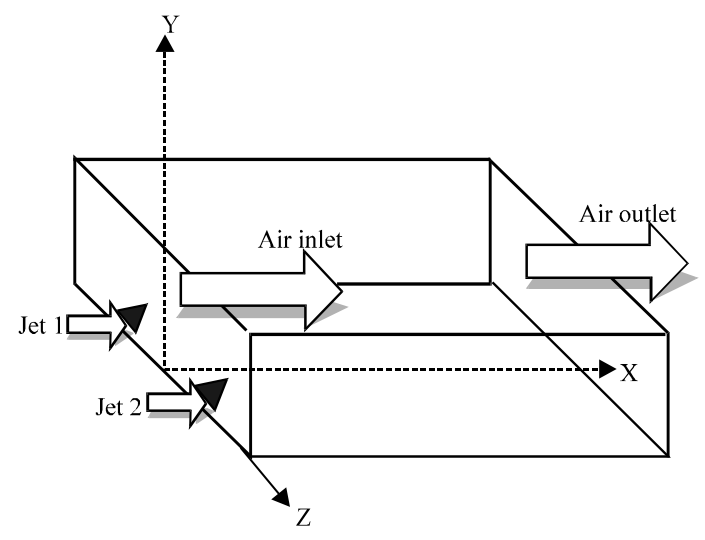

Fig. 3: Domain of calculation

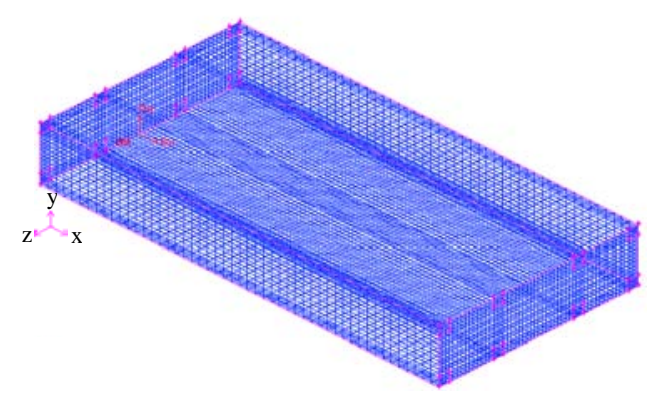

Fig. 4: Domain of grid calculation

across two square holes sections of dimension $(5 \times 5 \mathrm{~mm})$. The principal flow (air inlet face $\mathrm{ADEF}$ ) is emitted longitudinally across a rectangular section of width $100 \mathrm{~mm}$ and of height $30 \mathrm{~mm}$. The distance between the centre of an air jet and the $(0 \mathrm{Y})$ axis is of $\mathrm{H}=20 \mathrm{~mm}$. In this study, the sign + in quote indicates a normalised quantity. The heights are normalised by $\mathrm{H}$ and the temperature gaps are normalised by the reference temperature gap $\left(\Delta \mathrm{t}_{\mathrm{ref}}\right)$.

The molecular effects being negligible in front of the turbulence, the relationship $\Delta \mathrm{T} / \Delta \mathrm{T}_{\mathrm{ref}}$ can be assimilated to a concentration $\mathrm{C}$ which will vary between 1 at emission and 0 at the infinity. $\Delta \mathrm{T}$ is the difference between the initial temperature of the principal flow and the temperature of jets at an instant $t$.

This domain of calculation has 6 faces: the faces $(\mathrm{ADEF}),(\mathrm{BCGH}),(\mathrm{ABCB}),(\mathrm{EFGH}),(\mathrm{ABHF})$ and (DCGE). The principal flow is introduced through the face (ADEF). Air comes out through the face (BCGH) from the domain. The faces (ADCB), (EFGH), (ABHF) and (DCGE) are walls which constitutes the boundaries of the domain. Hot ait jet is introduced in the domain by two squares which are focused in the same plan as face (ADEF) on axe $(0 \mathrm{Z}+$ ) for its portion and others at the origin of distance $40 \mathrm{~mm}$ and materialise by jet 1 and 2 . 
Condition of calculation: At the input of the domain (entry of jet and of air), researchers have imposed the velocity conditions and at the output researcher are imposed the pressure conditions.

In this research, the chosen pressure is the atmospheric pressure. The value of the velocity imposed at the input for air is $5 \mathrm{~m} \mathrm{sec}^{-1}$ and for jets, three values have been set successively: 5,10 and $15 \mathrm{~m} \mathrm{sec}^{-1}$. The mesh is realised with the Gambit program. It is a regular grid type with its cells in the quadrilateral form. This is shown in Fig. 4.

\section{RESULTS AND DISCUSSION}

The numerical results show the influence of the initial jet velocity on their thermal fields at different positions. Figure 5-7 show the iso-values of the average temperature at positions $\mathrm{X}+=0.5$ and 5 for a longitudinal flow velocity of $5 \mathrm{~m} \mathrm{sec}$ and for different jet velocities $\left(5,10,15 \mathrm{~m} \mathrm{sec}^{-1}\right)$ where the Reynolds number are $\left(\mathrm{Re}=\mathrm{U}_{\mathrm{j}} \mathrm{d} / v\right)$ corresponding to $1200,2400,4800$. These results were obtained from a mesh size of 79628 meshes.
For more precisions, the mesh size was tied at the output of the jets. The under relaxation factors for the pressure, the turbulent kinetic energy, the turbulent dissipation ratio, the quantity of movement and energy are $0.3,0.8$, $0.8,0.7$ and 0.8 . On the $(0 \mathrm{X}+)$ axis, the scanning is carried out between $\mathrm{X}+=0$ and 10 on the $(0 \mathrm{Y}+)$ axis the scanning is carried out between $\mathrm{Y}+=0$ and 1.5 and on the $(0 \mathrm{Z}+)$ axis, the scanning is carried out between $Z+=-2.5$ and 2.5. In these figures, researcher notice that at $\operatorname{Re}=1200$, the jets are stalled on the plate under the influence of the principal flow mean while for $\mathrm{Re}=2400$ and 4800 , the jets are removed from the plate and gradually under goes the influence of the longitudinal flow. The zone at which the temperature is maximum (red zone), there is decrease with increase in Reynolds number when evolve from position $\mathrm{X}+=0.5-5$. This is as a result of the presence of whirl which turns to render the jets more energetic and favours its dilution in the principal flow. The experimental results are shown on Fig. 8-10 by using the iso-values of temperature. The profiles of the maximum temperature shown at Fig. 1a-c represent the comparison of numerical results to the experimental

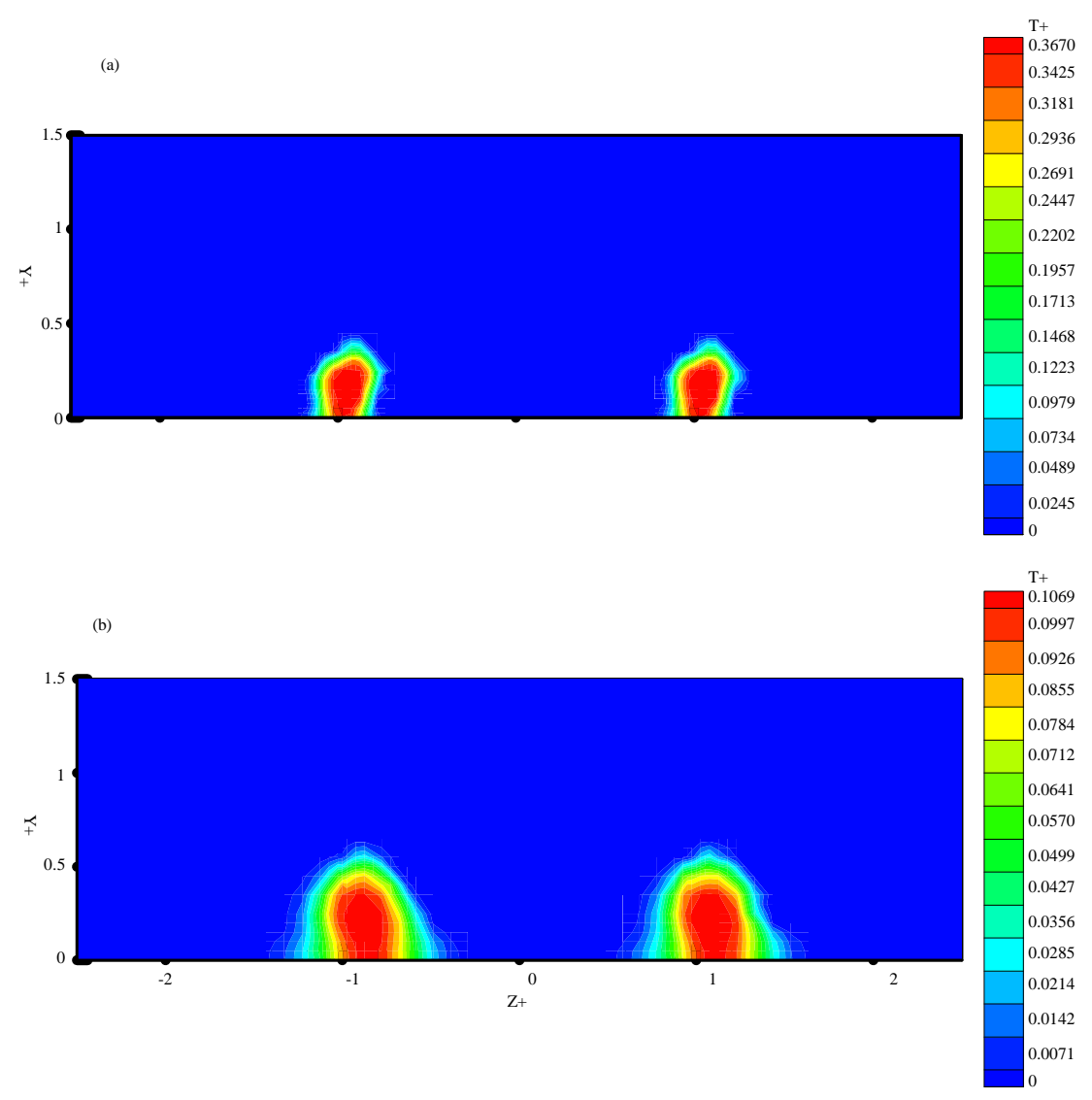

Fig. 5: Iso-values of dimensionless average temperature $\mathrm{Re}=1200 ; \mathrm{a}$ ): $\mathrm{X}+=0.5 ; \mathrm{b}$ ): $\mathrm{X}+=5$ 


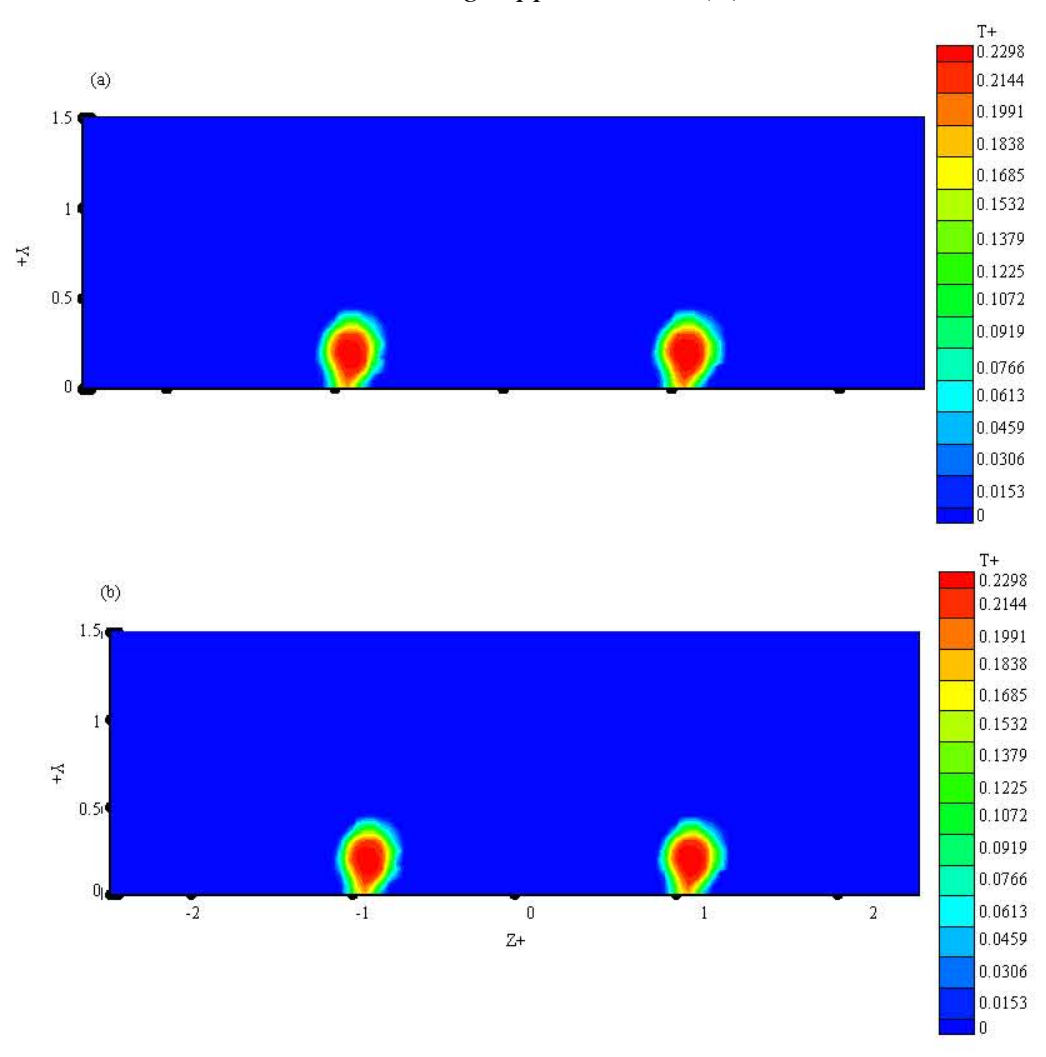

Fig. 6: Iso-values of dimensionless average temperature $\operatorname{Re}=2400 ; a)$ : $X+=0.5 ; b): X+=5$
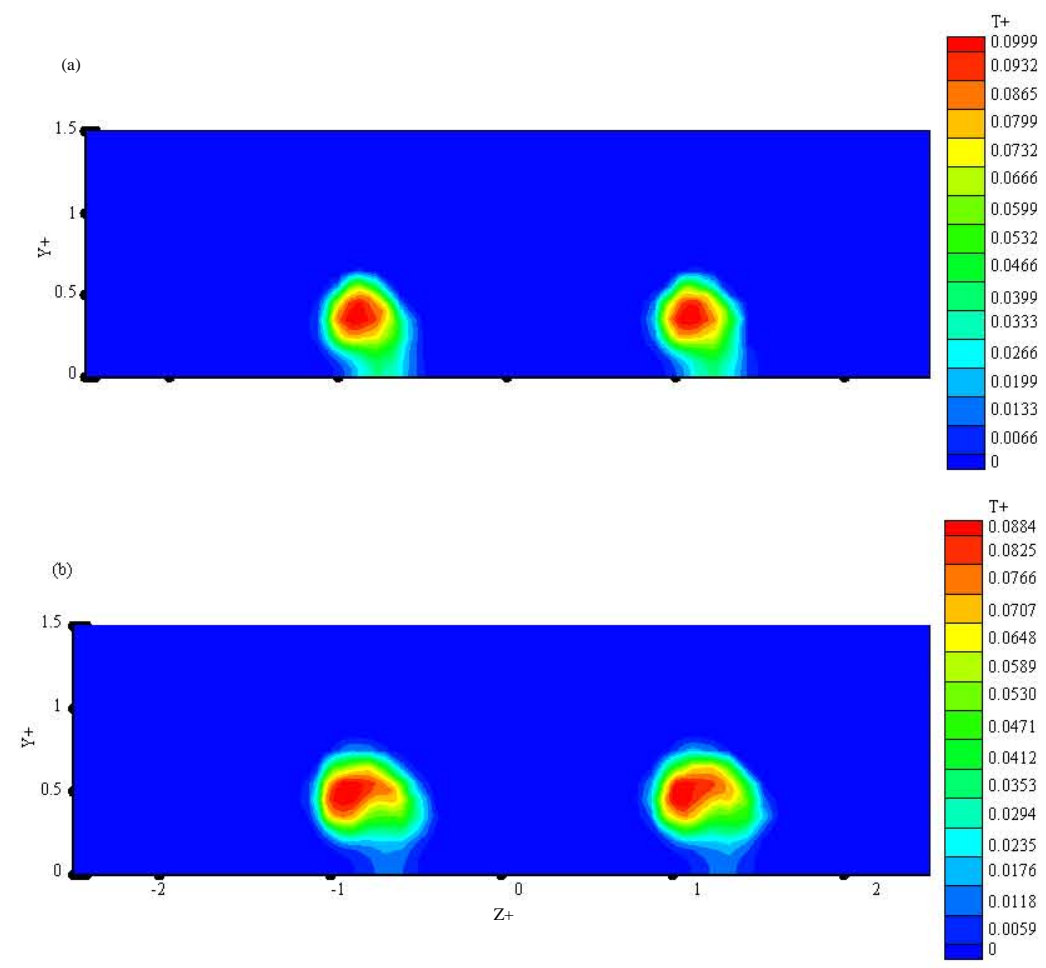

Fig. 7: Iso-values of dimensionless average temperature $\mathrm{Re}=4800 ; \mathrm{a}): \mathrm{X}+=0.5 ; \mathrm{b}): \mathrm{X}+=5$ 


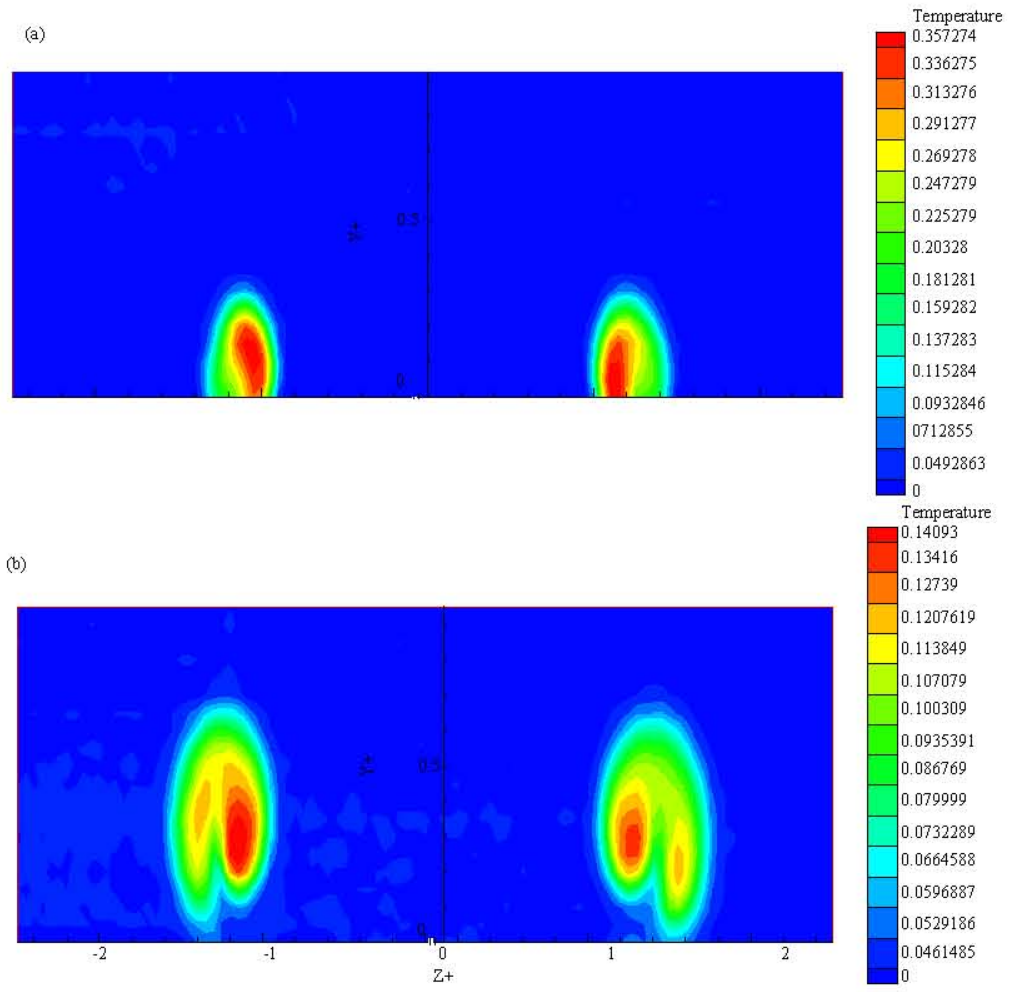

Fig. 8: Iso-values of dimensionless temperature $\operatorname{Re}=1200 ; a): X+=0.5 ; b): X+=5$
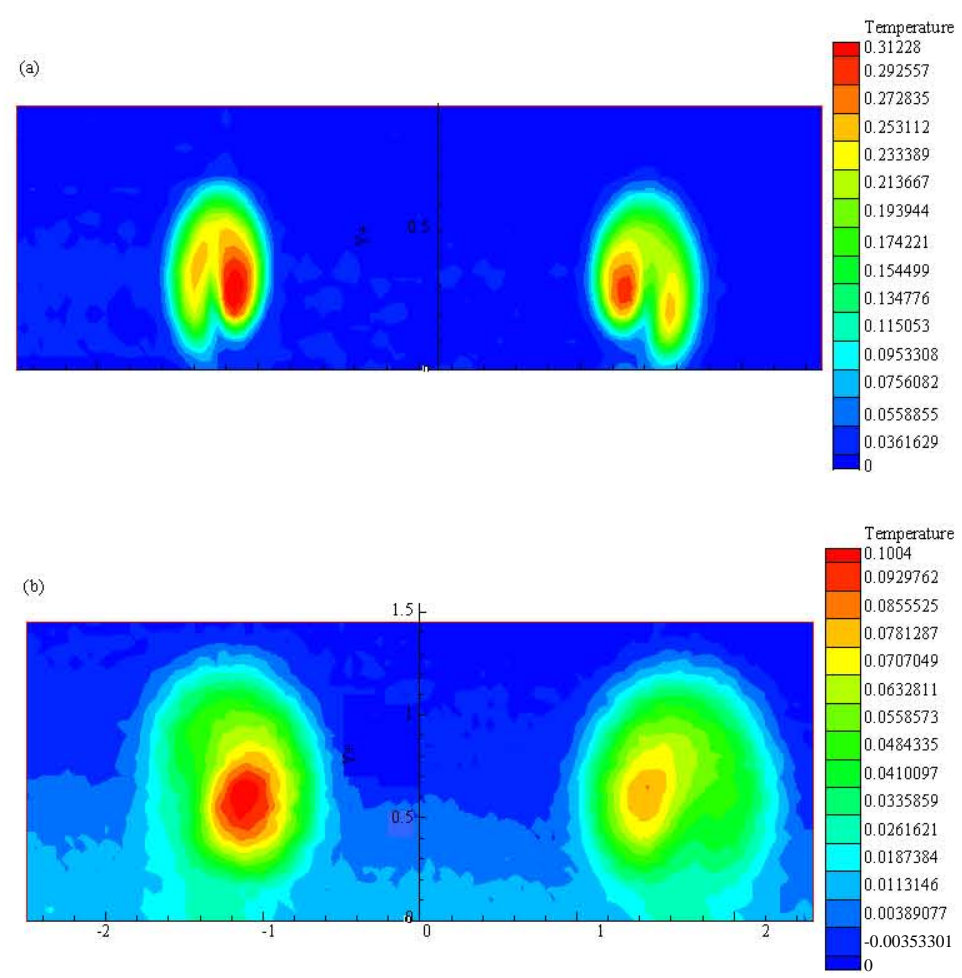

Fig. 9: Iso-values of dimensionless temperature $\operatorname{Re}=2400 ; a): X+=0.5 ; b): X+=5$ 


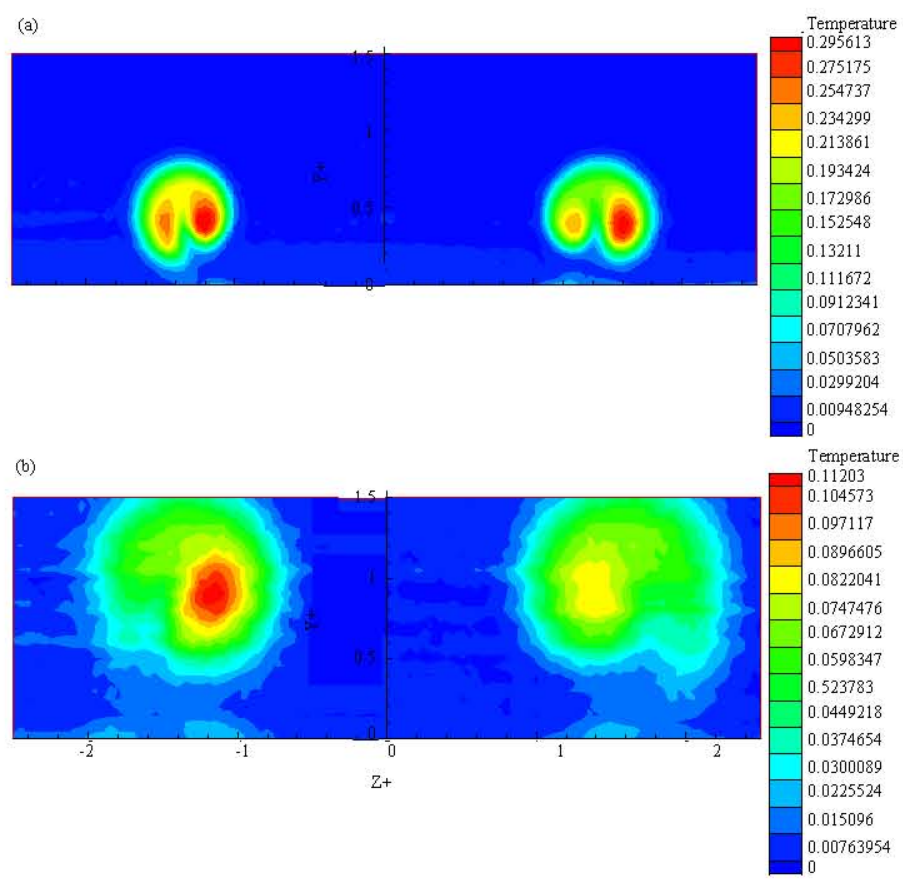

Fig. 10: Iso-values of dimensionless temperature $R e=4800 ; a): X+=0.5 ; b): X+=5$

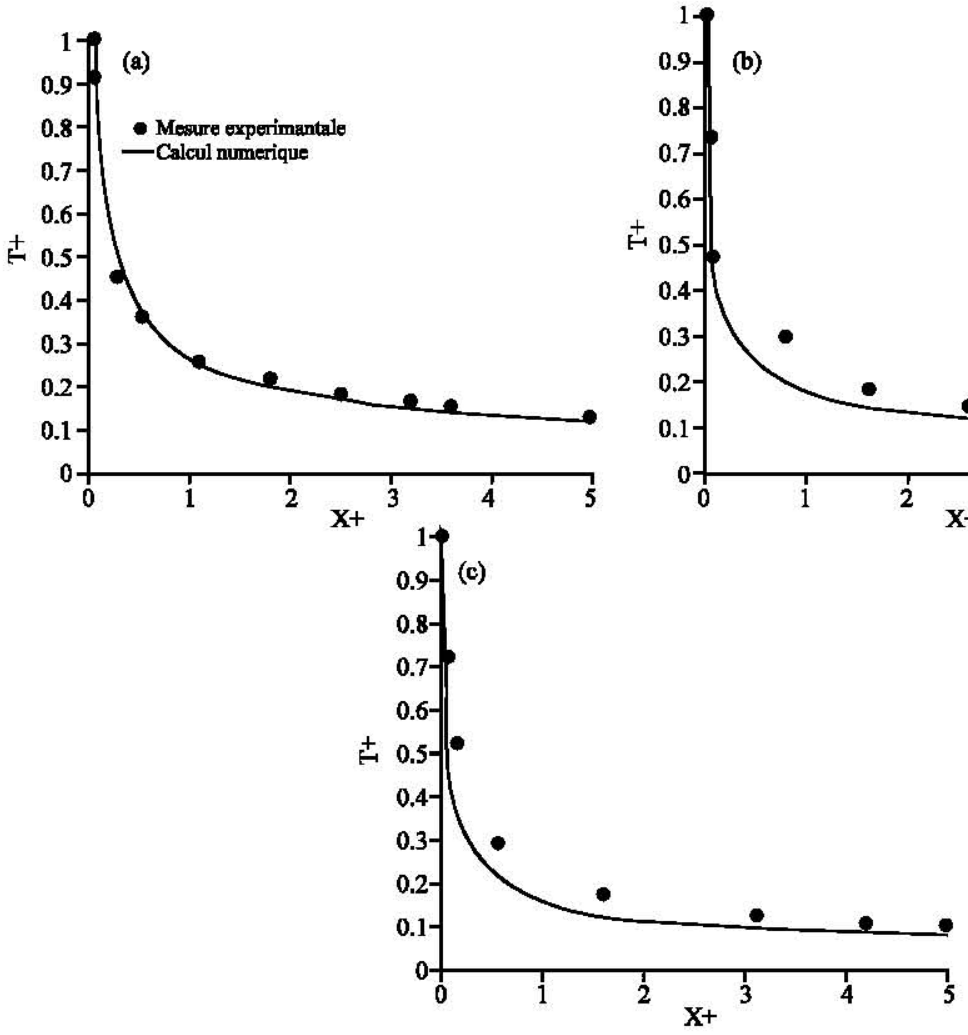

Fig. 11: Comparison of maximum temperature profiles obtained by numerical simulations and experimental measurement at: a) $\operatorname{Re}=1200 ;$ b) $\operatorname{Re}=2400$; c) $\operatorname{Re}=4800$ 
measurement. Researchers see that the different between the numerical calculation and the experimental measurement is higher at $\operatorname{Re}=4800$ than at $\operatorname{Re}=2400$ and 1200 at $\mathrm{X}+=0$ and 2.5 . This difference turns to cancel at $\mathrm{X}+=0$ and 5 positions. This is as a result of the turbulent nature of the flow. Researchers also notice that the drop in temperature is severe when we evolve from $\mathrm{X}+=0-2$. This is when the Reynolds number is high but in the case where the Reynolds number is low, it spreads rapidly.

\section{CONCLUSION}

In this research, researcher notice that the scalar temperature drops severely when passed from position $\mathrm{X}+=0-2.5$ and as well turn close to the temperature of the principal flow at $\mathrm{X}+=5$. Movers, this temperature drops increases with increase in the Reynolds number. The numerical solution has the same evolution as the experimental solution. The difference between these two solutions is their increase in Reynolds number. It springs also that at $\operatorname{Re}=1200$, the scalar under goes influence due to the principal flow and slacks on the plate mean while at $\operatorname{Re}=2400$ and 4800 , the scalar under goes less influence resulting from the principal longitudinal flow and unstitching the plates.

The temperature of the hottest zone drops with increase in the Reynolds number. This is due to the turbulent nature of the flow which renders the jet more energetic and this favouring its dilution in the principal flow (Gosse et al., 2006a, b). The approximation of the $\mathrm{k}-\varepsilon$ Models since the researches of Hunt and Vassillicos (1991) and Phillips (1991), researchers know that turbulent flow is the seat for a range of scale.

Researchers have approximated with numerical tools the experimental results. The concordance by comparison, researchers estimate that of calculation is satisfactory. Lets precise that the ameliorations of the temperature fields can be done by passing from the $\mathrm{k}-\varepsilon$ Model to LES (Large Eddy Simulation). The LES permits the calculations of big structures all by conserving a statistical representation of the smallest fluctuations. These calculations made stationary can also be realised in stationary with the aim of studding the behaviour of the scalar in time.

\section{ACKNOWLEDGEMENT}

The researchers acknowledge the CORIA UMR 6614 CNRS University of Rouen-France and The University of Ngaoundere, Cameroon.

\section{NOMENCLATURE}

\author{
Small letters: \\ $\mathrm{x}=$ Longitudinal coordinat $(\mathrm{m})$ \\ $\mathrm{y}=$ Vertical coordinat $(\mathrm{m})$ \\ $\mathrm{z}=$ Transversal coordinat $(\mathrm{m})$
}

\section{Capital letters:}

$\mathrm{H}=$ Distance separating one of the two holds from the principal axe $(\mathrm{m})$

$\mathrm{P} \quad=$ Pressure $(\mathrm{Pa})$

$\mathrm{T}=$ Temperature difference $(\mathrm{K})$

$\Delta \mathrm{t}_{\text {réf }}=$ Temperature difference between hot jet and the exterior $(\mathrm{K})$

$0 \mathrm{x}=$ Longitudinal axis

Oy $=$ Vertical axis

$\mathrm{Oz}=$ Transversal axis

\section{Greek symboles:}

$v=$ Kinetic viscosity of air $\left(\mathrm{m}^{2} \mathrm{sec}^{-1}\right)$

$\mu=$ Dynamic viscosity of air (Pa sec)

$\varepsilon=$ Dissipation ratio of the turbulent kinetic energy

$\mathrm{K}=$ Turbulent kinetic energy $\left(\mathrm{J} \mathrm{kg}^{-1}\right)$

$\rho \quad=$ Volumic mass $\left(\mathrm{m}^{3} \mathrm{sec}^{-1}\right)$

\section{No dimensional numbers:}

$\mathrm{Re}=\mathrm{Re}$ Reynolds number

$\mathrm{Pr}_{\mathrm{t}}=$ Prandtl turbulent number

\section{Exponents, indices and specials characters:}

+ Grandeurs adimensionnees (par $\mathrm{H}$ pour les longueurs) et (par $\Delta \mathrm{T}_{\text {ref }}$ pour les ecarts de temperatures):

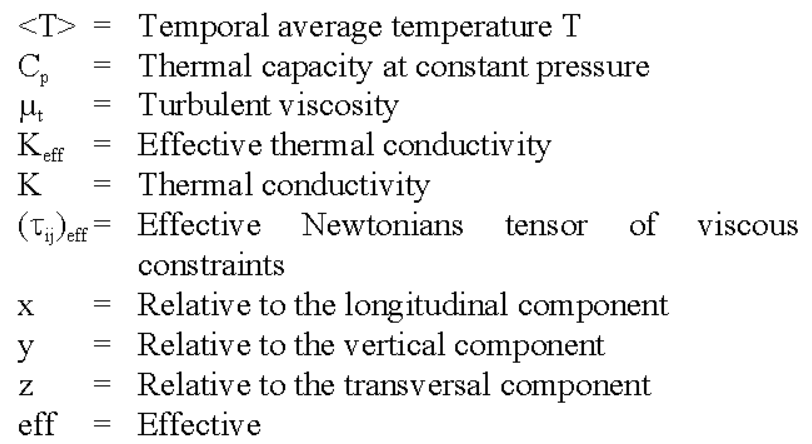

\section{REFERENCES}

Bray, T.P. and K.P. Garry, 1999. Optimisation of jet vortex generators with respect to system design parameters. Aeronaut. J., 103: 475-479.

Gosse K., P. Paranthoen, B. Patte-Rouland and M. Gonzalez, 2006a. Dispersion in the near wake of an idealized car model. Int. J. Heat Mass Trans., 49: 1747-1752. 
Gosse, K., Patte-Rouland, B., M. Gonzalez and P. Paranthoen, 2006b. Scalar dispersion in the near wake of a simplified model car. Exp. Fluids, 40: $135-140$.

Hunt, J.C.R. and J.C. Vassillicos, 1991. Kolmogorov's Contributions to the Physical and Geometrical Understanding of Small Scale Turbulence and Recent Developments, Turbulence and Stochastic Process. Published Royal Society, London.

Kothnur, P.S. and N.T. Clemens, 2005. Effects of unsteady strain rate on scalar dissipation structures in turbulent planar jets. Phys. Fluid., 17: 104-125.
Phillips, O.M., 1991. The Kolmogorov Spectrum and its Oceanic Cousins: A Review, Turbulence and Stochastic Processes. Published Royal Society, London.

Shih, T.H., J. Zhu and J.A. Lumley, 1995. New reynolds stress algebraic equation model. Comp. Meth. Applied Mech. Eng., 125: 287-302.

Vincont, J.Y., S. Simoens, M. Ayrault and J.M. Wallace, 2000. Passive scalar dispersion in a turbulent boundary layer from a line source at the wall and downstream of an obstacle. J. Fluid Mech., 424: $127-168$. 